\title{
How does the ecological foraging behavior of desert kangaroo rats (Dipodomys deserti) relate to their behavior on radial mazes?
}

\author{
WILLIAM TIMBERLAKE and CYNTHIA M. HOFFMAN \\ Indiana University, Bloomington, Indiana
}

\begin{abstract}
Experiment 1 showed that laboratory-reareddesert kangaroo rats, like domestic Norway rats, efficiently search for food on a radial arm maze (RAM) by avoiding revisiting arms within a trial. By placing an RAM on the floor so the animals could approach food from any direction, Experiment 2 tested whether efficient search by kangaroo rats was based on tactics of distance minimizing, central-place foraging, trail following, or meandering. In contrast to the dominant trail-following tactic of domestic Norway rats (Hoffman, Timberlake, Leffel, \& Gont, 1999), kangaroo rats tended to distance minimize, whether maze arms were present or not. Experiment 3 indicated that kangaroo rats treated a floor configuration of eight food cups as two patches of four, based on beeline travel between patches and meandering within them. We conclude that similar performance in an elevated RAM by different species can be based on different tactics, and we suggest that a laboratory apparatus can be used to cast light on nicherelated mechanisms.
\end{abstract}

Desert kangaroo rats (Dipodomys deserti) inhabit regions characterized by deep sand dunes and sparse vegetation. They are primarily granivorous, although they also forage for green vegetation and insects. Using their front paws, kangaroo rats collect visible seeds and sift surface sand for others $(75 \%$ of accessible seeds are found on or within $2 \mathrm{~mm}$ of the surface; Reichman, 1984). They carry the collected seeds in external cheek pockets to their burrow, where they store them in one or two larder hoards (Stern, 1980). Patches of seeds occur under fruiting bushes, as well as in the open, where dense clumps are created by the interaction of wind and water with the terrain. Seed patches are frequently stable over time (Reichman, 1984; Reichman \& Oberstein, 1977); patches in the open are less stable but may be denser than patches under bushes (Reichman \& Oberstein, 1977).

Given the patchy distribution of seeds in the habitat of desert kangaroo rats, most researchers have assumed that kangaroo rats are patch specialists that focus their foraging on high-concentration seed patches (Kotler, 1984, 1985; Stern, 1980; Thompson, 1982). Data from field observations appear to support this view. Thompson observed that foraging desert kangaroo rats alternate between rapid, direct long-distance movements (beelines) and short, meandering moves interrupted by frequent sifting. During a

National Science Foundation Grants IBN9408366 and 9817175 to W.T. supported this work. C.M.H. was supported by National Research Service Award 1F32MH10662. The authors thank Tanya Houghton for considerable help with the experiments and Joe Leffel for his invaluable technical assistance. Correspondence should be addressed to W. Timberlake, Department of Psychology, Indiana University, 1101 E. 10th St., Bloomington, IN 47405 (e-mail: timberla@indiana.edu). foraging bout, they initially visit four or five patches before returning to the burrow and depositing collected seeds; then they exit again to visit another four or five patches before returning once more (Thompson, personal communication to C.M.H., June 24, 1992), a multiple patch form of central-place foraging. Also, according to Thompson, desert kangaroo rats regularly visit a set of patches for a period of several months, supporting the importance of memory for spatial locations.

The present experiments had two related purposes: to determine whether the foraging patterns of desert kangaroo rats observed in the field could be shown and manipulated in a standardized laboratory environment and to clarify evolved (niche-related) mechanisms potentially underlying both field and laboratory behavior. We began with the traditional ethological assumption that the behavior of animals under appropriate artificial circumstances can be related to the niche-related mechanisms that determine behavior in natural settings (Timberlake, 2001a, 2002; Tinbergen, 1951). Ethologists have often manipulated the characteristics of artificial releasing stimuli in controlled settings in order to analyze the perceptual-motor mechanisms underlying field behavior. For example, Tinbergen and Perdeck (1950) presented gull chicks with visual models of adult gull beaks and heads in order to discover which characteristics produced the most reliable and vigorous pecking (see also Hailman, 1967).

Timberlake (2001a, 2002) pointed out that laboratory scientists often use a variant of this ethological approach in the process of developing laboratory tasks. Laboratory scientists manipulate (tune) aspects of the seemingly artificial apparatus and procedures in order to discover characteristics that determine reliable and vigorous respond- 
ing. However, instead of focusing on what these characteristics might reveal about the perceptual-motor and motivational processes of the animal, most laboratory scientists use the tuned apparatus and procedures to test the relation of abstract response measures to independent variables. For example, instead of using the autoshaping procedure in pigeons (the presentation of a keylight predicting grain delivery; see Hearst \& Jenkins, 1974) to analyze the ecological relevance of sensory characteristics of the laboratory stimuli that produce pecking, laboratory researchers used the procedure to test general hypotheses about its relation to Pavlovian conditioning and the role of predictability in learning.

In short, if we assume that experimenters build nicherelevant characteristics into their apparatus and procedures by tuning experiments to produce vigorous and interpretable responding, two general conclusions follow. First, it should be possible to use systematic variation of traditional laboratory paradigms to explore the contribution of niche-related mechanisms to the laboratory behavior of a particular species. At the same time, it should be possible, by manipulating the apparatus and procedure, to engage and analyze the mechanisms underlying their behavior in the field.

On the basis of this viewpoint, the present experiments had two purposes. The first was to examine how the desert kangaroo rat, a patch-foraging species not unlike the Norway rat, dealt with the spatial-learning problem posed by the radial arm maze (RAM), an apparatus developed with Norway rats. The second purpose was to analyze more specifically the mechanisms the kangaroo rats used to solve spatial problems of the sort posed by the RAM environment. In Experiment 1, we tested whether desert kangaroo rats were able to map components of their nicherelated foraging behavior and mechanisms onto an RAM designed for Norway rats, sufficiently well to search the maze efficiently. In Experiment 2, we examined the specific foraging tactics used by the kangaroo rats (as compared with Norway rats) in dealing with a RAM first by placing the apparatus flat on the floor of an arena (thereby removing the path constraints of the arms) and then by removing the maze arms entirely (thereby removing the arms as guides or trails). In Experiment 3, we explored how the distribution of food cups in an arena might map onto foraging mechanisms and behavior by examining whether artificial patches would produce the combination of beeline locomotion between patches and meandering search within patches that has been reported in the field.

\section{EXPERIMENT 1}

The purpose of the first experiment was to determine whether desert kangaroo rats (Dipodomys deserti) would show efficient spatial foraging on a typical baited RAM. Efficient search of an RAM in a Norway rat is usually shown by their selection of seven or more novel arms within the first eight choices of a trial (in other words, an average of only one arm revisited within the first eight choices). The field observations of Thompson (1982), which showed that kangaroo rats frequently efficiently visit a similar set of spatially distributed patches each night, indicated that they are capable of using spatial reference memory across nights and effective working memory within a night.

In the laboratory, Langley (1994) confirmed the ability of desert kangaroo rats to accurately remember spatial locations where they found food. Kangaroo rats that had found a plastic token related to food located in one buried box accurately dug for a token hidden in the same location in a second box. Accurate spatial reference memory for food has also been shown in the closely related Merriam's kangaroo rat (D. merriami) by Jacobs (1992) and Rebar (1995). However, we do not know of any data on kangaroo rats tested in a standard laboratory spatial working memory task, such as the elevated RAM.

On the basis of their apparent ability to forage efficiently within a night on multiple patches in the wild, we anticipated that kangaroo rats would perform well on an RAM. However, we had little direct evidence about their working memory. In fact, Olton, Handelmann, and Walker (1978) offered a specific alternative prediction. These researchers attributed the RAM performance of Norway rats to a win-shift foraging strategy. A win-shift strategy is one in which a forager, having found and eaten food at one site, travels to different sites in subsequent forays during the foraging bout. Because kangaroo rats appear to have evolved to forage for spatially separated high-concentration seed patches that cannot be depleted in one visit, Olton et al. suggested that they were more likely than Norway rats to be win-stay foragers. In other words, on the basis of niche-related mechanisms evolved in an environment of concentrated patches, kangaroo rats on an RAM should be more likely than Norway rats to repeat arm visits during a particular trial (despite never having received a reward on repeat visits).

In the present experiment, desert kangaroo rats were tested on a standard eight-arm baited RAM to determine how efficiently they searched the maze. The arms of a typical RAM radiate from a central platform at equal angles. At the beginning of a trial, the end of each arm is baited with a single piece of food, and the rat is then placed on the central platform and allowed to make 12 choices. The most efficient procedure is to collect all the food while visiting each arm only once. Norway rats (Rattus norvegicus) gather food on an RAM with great proficiency after very few trials, selecting arms well above chance level. To the extent that the RAM engages patch-related foraging mechanisms in Kangaroo rats as well, we expected similar efficiency in them.

We report the percentage of novel arms visited per trial as a measure of the kangaroo rats' ability to avoid revisits to an arm within a trial. We also report the percentage of visits to an adjacent arm, to assess whether the kangaroo rats avoid revisits by using a simple response algorithm within a trial, like choosing an adjacent arm, rather than remembering individual spatial locations already visited. 
Olton and Samuelson (1976) concluded that Norway rats did not depend on such a response algorithm or on scent marking to perform accurately on the RAM. By similar reasoning, if desert kangaroo rats also perform efficiently on the RAM and do not use a response algorithm or scent mark, we will conclude that they also use spatial memory to forage effectively.

\section{Method}

Subjects. Eight adult desert kangaroo rats (Dipodomys deserti) were tested, 4 males and 4 females. The animals were related to a group of Kangaroo rats trapped with Sherman traps $42 \mathrm{~km}$ east of Twentynine Palms (San Bernadino, CA) in May 1994 by C.M.H. She held a California Fish and Game student trapping permit. This site is on stabilized sand dunes and is dominated by creosote (Larrea tridentate) and bunch grass (Hilaria rigida). The kangaroo rats were trapped from the same population as that observed by Thompson (1982). All the kangaroo rats were adults when captured; their weights ranged from 90 to $140 \mathrm{~g}$. Seven of the subjects in the present experiment were first-generation laboratory descendents of the wild-caught animals; the remaining male was one of the smaller wild-caught males. We did not expect notable sex differences in the foraging of these animals, because of similarities in size and their relatively monogamous mating system, but we included both males and females to form a more representative small sample of the population.

The kangaroo rats were housed in the Animal Behavior laboratory at Indiana University from the time of birth or capture through the completion of all the experiments reported here. The kangaroo rats were housed individually in $38 \times 30 \times 18 \mathrm{~cm}$ cages. An $18 \times 30 \times$ $18 \mathrm{~cm}$ stainless steel box, open at the bottom, provided a shelter in which the kangaroo rats spent the hours when room lights were on. The dark phase of the animal's 10:14-h light:dark cycle began at $1200 \mathrm{~h}$. This partially reversed light:dark cycle was set by bright white overhead room lights. In addition, six 40-W soft-light red bulbs remained on for $24 \mathrm{~h}$ a day. Cages were filled with fine river sand to a depth of about $4 \mathrm{~cm}$, which allowed the kangaroo rats to sand bathe in the cage and cache seeds in the nest chamber.

Before the experiment began, twice each week, the kangaroo rats were provisioned with $70 \mathrm{~g}$ of mixed birdseed (containing millet, milo, and sunflower seed) and a 6-g Romaine lettuce leaf. This provisioning allowed them to feed ad lib. Kangaroo rats are able to get necessary water from the seeds ( $40 \%$ water by weight) and lettuce and do not require access to free water. During the course of the experiments, the kangaroo rats continued to receive mixed seed in their home cages as usual, but only one 6-g leaf of lettuce per week at the end of each week's testing. The remaining lettuce could be obtained on the maze. The 1 subject that never collected the lettuce on the maze was given $10 \mathrm{~g}$ of lettuce in its home cage at the end of each week's testing. Testing was conducted between 2 pm and 6 pm 4 days a week.

Apparatus. We used an eight-arm radial maze constructed of wood with thin Masonite edges. The $10 \times 70 \mathrm{~cm}$ arms were attached to the sides of the octagonal central platform, $14.5 \mathrm{~cm}$ on a side and $34 \mathrm{~cm}$ across between parallel sides. Each arm was fitted with hard fiberboard side walls. The right side wall was $3 \mathrm{~cm}$ high. The first $32-\mathrm{cm}$ section of the left side wall was $12 \mathrm{~cm}$ high, and the remainder was $3 \mathrm{~cm}$ high. A 2.5 -cm-diameter, 2 -cm-high cup, milled from aluminum stock, was placed $4 \mathrm{~cm}$ from the end of and equidistant from the sides of the arm. The entire apparatus was painted gray and was located in a quiet experimental room, $6.30 \times 3.24 \mathrm{~m}$.

The kangaroo rats were transported to and from the staging area inside the experimental room in their individual cages. Two cages at a time were placed inside a lidded cardboard box in the colony room, the box was covered with brown felt, and the kangaroo rats were wheeled to the experimental room on a cart. Once inside the experimental room, both cages were removed from the box and placed on a rack in a consistent location each day. The experimental room was dimly lit with five $40-\mathrm{W}$ soft-light red bulbs. The light was sufficient for the experimenter to observe the kangaroo rat and to record the data. The experimenter sat in a chair $1 \mathrm{~m}$ away from the maze.

Procedure. Each kangaroo rat was tested one trial a day, 4-5 days per week. Two $2 \times 2 \mathrm{~cm}$ squares of Romaine lettuce were placed in each food cup prior to the beginning of each trial. We used lettuce on the RAM because kangaroo rats collect it as they do seeds while foraging in the desert and because it is a highly effective reward for kangaroo rats performing laboratory tasks. Furthermore, to use seed as a reward, we would have had to precisely restrict the daily provision of seed for each kangaroo rat to just the amount necessary to maintain its body weight, instead of being able to provide each with enough seed to maintain a larder in its home cage.

At the beginning of each trial, the kangaroo rat was placed in the center of the maze, oriented away from the experimenter. A trial ended when the kangaroo rat had visited each of the eight cups once and one additional minute had elapsed, when it had visited the cups a total of 12 times, or when 5 min had elapsed, whichever came first. During the trial, the experimenter recorded the cups visited and the time elapsed at the end of the trial. At the end of the trial, the kangaroo rat was gently corralled into a carrying cage and was returned to its home cage. We then recorded whether lettuce remained in any food cups that had been visited on that trial. On those few occasions on which a kangaroo rat jumped off of the maze onto the floor during a trial, the experimenter gently corralled the kangaroo rat into a carrying cage and placed it back on the maze until the end of the trial. After the first few days, no kangaroo rats jumped off the maze.

The kangaroo rats were always tested individually, but for counterbalancing purposes, they were run in groups of 4, alternating by days which group was run first. To decrease the likelihood that the kangaroo rats would be influenced by odor cues left on the maze by a previous kangaroo rat, the maze was wiped with Roccal-D (a disinfectant, detergent, and deodorant) before each kangaroo rat was tested.

To assess whether the kangaroo rats searched efficiently, we compared the percentage of novel arms visited in the first eight cup visits with chance. The percentage of novel cups visited by chance on an elevated eight-arm maze was 5.25 (given no memory of previously visited cups). Chance for novel arms entered in eight choices was calculated with the formula $N-N[(N-1) / N]^{N}$, where $N$ is the number of arms.

To perform well, the kangaroo rats could remember and not return to the arms they had already visited on a trial, or they could use a response algorithm, such as "always enter the arm on the left," when they returned to the center of the maze. To determine whether the kangaroo rats used such a response algorithm, we calculated the percentage of visits to adjacent arms by dividing the total visits to adjacent arms by the total number of visits on each trial.

Dependent variables were analyzed with $t$ tests and analyses of variance (ANOVAs). Data were combined into two-trial blocks for analysis. One-group, one-tailed $t$ tests were conducted when the performance of the kangaroo rats was compared with chance or with the expected value for an optimal forager. Tests with predictability of less than .05 are reported as significant.

\section{Results}

The kangaroo rats tended to visit each arm on the maze once during their first eight choices. The left side of Figure 1 shows a rapid increase in the percentage of novel cups visited per trial $[F(7,49)=10.6, p<.01]$, and the average percentage of novel cups visited in the first eight choices was significantly above chance, tested over all tri- 

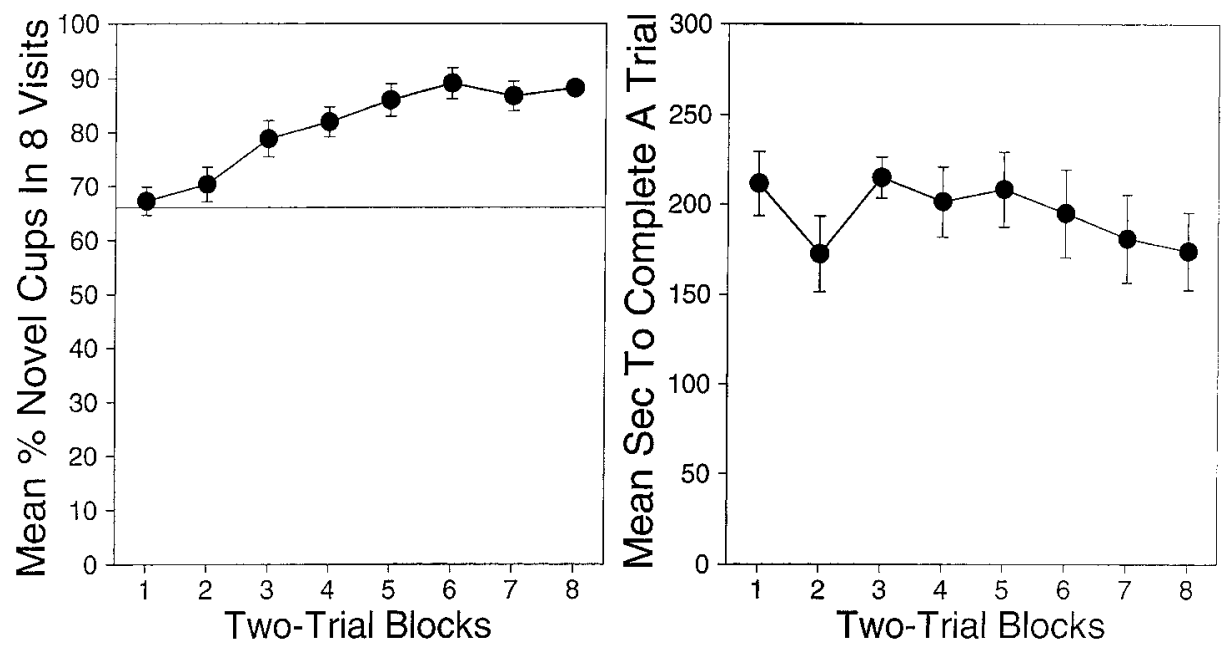

Figure 1. Mean kangaroo rat performance on a standard elevated radial arm maze. Left: novel arms entered in the first eight visits averaged over two-trial blocks; the line across the figure represents the percentage of novel arms chosen in the first eight visits that would be expected by chance (5.25/8). Right: time to complete a trial in seconds (eight choices or $5 \mathrm{~min}$ ) averaged over two-trial blocks (data are from Experiment 1).

als $[t(7)=10.46, p<.01]$. The right side of Figure 1 shows a modest but significant decline in the amount of time taken to complete each trial $[F(7,49)=2.40, p<.05]$.

Table 1 shows that the percentage of visited food cups from which lettuce was taken was initially low but increased significantly over trials $[F(7,49)=3.38, p<.01]$. The rats typically either pouched both pieces of lettuce or took neither. A comparison of Table 1 with the left side of Figure 1 shows that the rise in percentage of rewards taken lagged considerably behind the emergence of efficient search. The animals clearly visited novel arms efficiently well before the point at which they took lettuce from more than $80 \%$ of the arms visited. Finally, Table 1 also shows that although the percentage of visits to an adjacent arm increased significantly over days $[F(7,49)=4.55, p<.01]$, they still averaged only $17.9 \%$ of adjacent arm choices per trial, indicating that the animals did not depend on a response algorithm for efficient search.

\section{Discussion}

In keeping with their apparent spatial abilities in the wild, the desert kangaroo rats searched an elevated RAM very efficiently, visiting novel arms with a high level of accuracy similar to that of Norway rats. Although desert kangaroo rats specialize in foraging on concentrated seed patches in the field, the present animals did not display the tendency toward a win-stay strategy on the RAM predicted by Olton et al. (1978). If their RAM choice behavior was based on natural-foraging mechanisms, the behavior of our desert kangaroo rats suggests two possible ways in which they treated the maze: Either they behaved as though each arm were the location of a single food item within a patch that included all the arms and followed a win-shift strategy among food items, or they behaved as though each arm were a separate patch and showed a win-shift strategy between patches. We will consider these possibilities further in Experiment 3.

Our data support the conclusion that desert kangaroo rats use spatial memory, and not a response algorithm, to avoid arm revisits within a trial on an elevated RAM. Although the percentage of visits to an adjacent arm increased over days, it averaged only $17.9 \%$ over all days. Thus, for kangaroo rats, the percentage of visits to an adjacent arm was lower than that typically found for Norway rats on the same maze (e.g., Timberlake \& White, 1990). We did not rule out the possibility that the kangaroo rats used olfactory marking, but we considered it unlikely on two grounds. First, they were never observed to body mark or leave deposits. Second, marking trails to patches is not a particularly useful strategy to evolve in a relatively solitary species that lives on a sandy substrate that, like the patches themselves, shifts as the wind blows.

Table 1

Mean Percentages of Rewards Taken and Visits to Adjacent Arms on the Elevated Radial Arm Maze

\begin{tabular}{|c|c|c|c|c|c|c|c|c|c|c|c|c|c|c|c|c|c|c|}
\hline \multirow[b]{2}{*}{ Measure } & \multicolumn{2}{|c|}{ Block 1} & \multicolumn{2}{|c|}{ Block 2} & \multicolumn{2}{|c|}{ Block 3} & \multicolumn{2}{|c|}{ Block 4} & \multicolumn{2}{|c|}{ Block 5} & \multicolumn{2}{|c|}{ Block 6} & \multicolumn{2}{|c|}{ Block 7} & \multicolumn{2}{|c|}{ Block 8} & \multicolumn{2}{|c|}{ Mean } \\
\hline & $M$ & $S E$ & $M$ & $S E$ & $M$ & $S E$ & $M$ & $S E$ & $M$ & $S E$ & $M$ & $S E$ & $M$ & $S E$ & $M$ & $S E$ & $M$ & $S E$ \\
\hline Percentage of rewards taken & 52.9 & 16.5 & 52.7 & 16.6 & 65.6 & 15.7 & 83.9 & 12.3 & 87.5 & 12.5 & 89.4 & 9.7 & 79.2 & 11.6 & 100.0 & 0.0 & 76.4 & $\overline{9.4}$ \\
\hline Percentage of visits to adjacent arms & 3.5 & 2.3 & 8.1 & 3.9 & 16.0 & 4.7 & 22.2 & 6.5 & 27.3 & 8.3 & 23.3 & 11.6 & 35.0 & 8.3 & 34.2 & 8.3 & 21.2 & 5.0 \\
\hline
\end{tabular}


The kangaroo rats on an RAM also showed several contrasts to the behavior of Norway rats. First, they did not appear to eat food rewards while on the maze; instead, they put the lettuce in their cheek pockets and ate it in their home cages at the end of the trial. Also in contrast to Norway rats, the kangaroo rats initially collected only $52.6 \%$ of the lettuce available at the food cups they visited, although by the end of the experiment, they collected $80 \%-$ $100 \%$ of the available lettuce. It follows that the kangaroo rats did not depend on consuming or even collecting a reward on each arm to remember which arms they had visited in a trial. It could be that the sight (or olfactory cues) of the lettuce served as a secondary reinforcer, but it is difficult to explain, solely on the basis of secondary reinforcement, why the kangaroo rats did not revisit arms to obtain the lettuce. It would be interesting to compare the effects of sight and olfaction of the reward versus access to the food, but one would need some unrewarded arms and a control group with all unrewarded arms to clarify the phenomenon of secondary reinforcement of maze search.

To us, the lack of dependence of efficient search by the kangaroo rats on actually obtaining the reward bears potential resemblance to the findings of Timberlake and White (1990) that rats that were run without food reward on an RAM still searched efficiently. This evidence of persistent efficient search in the absence of a reliable primary reward (the kangaroo rats) or of any reward (the Norway rats) appears to require a redefinition of the term win (as in win-shift) if that concept is to be used as a causal construct. Most important, it indicates a possible rewardindependent specialization in patch-foraging rodents for efficient locomotor search of a patch (see also Tinsley, Rebec, \& Timberlake, 2001, for the ability of a dopamine D1 receptor agonist in rats to facilitate efficient maze search in the absence of any food).

\section{EXPERIMENT 2}

On the basis of Experiment 1, we conclude that desert kangaroo rats, like Norway rats, use working spatial memory to avoid revisits to maze arms on an elevated RAM. Most researchers have followed Olton et al. (1978) in presuming that efficient RAM search is based on a strategy for employing working memory called win-shift (when you find a reward, you do not revisit until you have looked everywhere else). However, that rats also readily show efficient search of an unbaited RAM (Timberlake \& White, 1990) suggests that their overall strategy is less win-shift than examine-shift. The similar performance of desert kangaroo rats in efficiently searching maze arms in Experiment 1, despite their pouching, rather than consuming, the reward on the maze and initially pouching only half of the rewards in visited arms, suggests a similar examineshift strategy.

A shift strategy by itself does not specify the pattern of choosing novel arms; it predicts only that animals will not revisit arms. To predict the pattern of visits in a trial, it is necessary to specify a tactic for implementing the shift strategy. Among the tactics that might be used are distance minimizing in choosing rewards, central-place foraging with the center of the maze as a temporary "base," trail following by treating arms as trails, and meandering randomly (without repeating choices). Unfortunately, in an elevated RAM, these tactics cannot be distinguished, because the animals are confined to the maze. So after each choice, they must pass back though the central platform and along another arm to approach a novel food cup. However, Hoffman, Timberlake, Leffel, and Gont (1999) and Timberlake, Leffel, and Hoffman (1999) showed that these tactics could be distinguished in Norway rats by using a floor RAM in which the center, arms, and food cups of a six-arm RAM were placed flat on the floor of an arena. By using a floor RAM, we can make a clear contrast of tactics, particularly by comparing predictions of foraging locomotion in the presence and absence of maze arms.

On a floor RAM, a distance-minimizing tactic predicts that animals, after traveling from the center to their first food cup, should move directly from one food cup to the next in a roughly circular pattern, regardless of the presence or absence of arms. A central-place foraging tactic focused on the central platform predicts that animals will exhibit a series of forays out to a food cup, followed by a return to the platform, again regardless of the presence of arms. A trail-following tactic implies that animals will follow arms (as trails) in moving from one food cup to the next. In the absence of arms, they will likely return to the arena wall (the closest available approximation to a trial) between food cups. Finally, a meandering tactic implies that the animals will pay little attention to the presence or absence of arms and, instead, will meander back and forth across the floor as they search for food (Thompson, 1982).

As can be seen in Figure 2, Norway rats on a floor RAM with (left) and without (right) maze arms at asymptote (Trials 11-12) showed a trail-following tactic, predominantly approaching food cups along the arms when arms were present. When arms were absent, the rats traveled along the walls of the arena (the box around the floor maze in Figure 2; Hoffman et al., 1999; Timberlake et al., 1999). Such behavior could be predicted on the basis of the marked tendencies of Norway rats in the wild to construct and predominantly follow trails in moving in and around colonies, even when searching for food (Calhoun, 1962; Telle, 1966).

On the basis of the foraging behavior of kangaroo rats in the wild, we would predict that, unlike Norway rats, the kangaroo rats will not show predominant trail following. Instead, in keeping with their ecology, they should show more distance minimizing and/or meandering. Distance minimizing would be predicted on the basis of their ability to use visual cues, such as saltbushes and bunch grass, to move between patches of seeds. Meandering could be predicted on the basis of their tendency to wander within a patch among different subpockets of seeds. Trail following to food would not be expected, because long-lasting trails are not maintained between food patches. Not only is there shifting sand, but desert kangaroo rats do not form multianimal social collectives and foraging groups that 

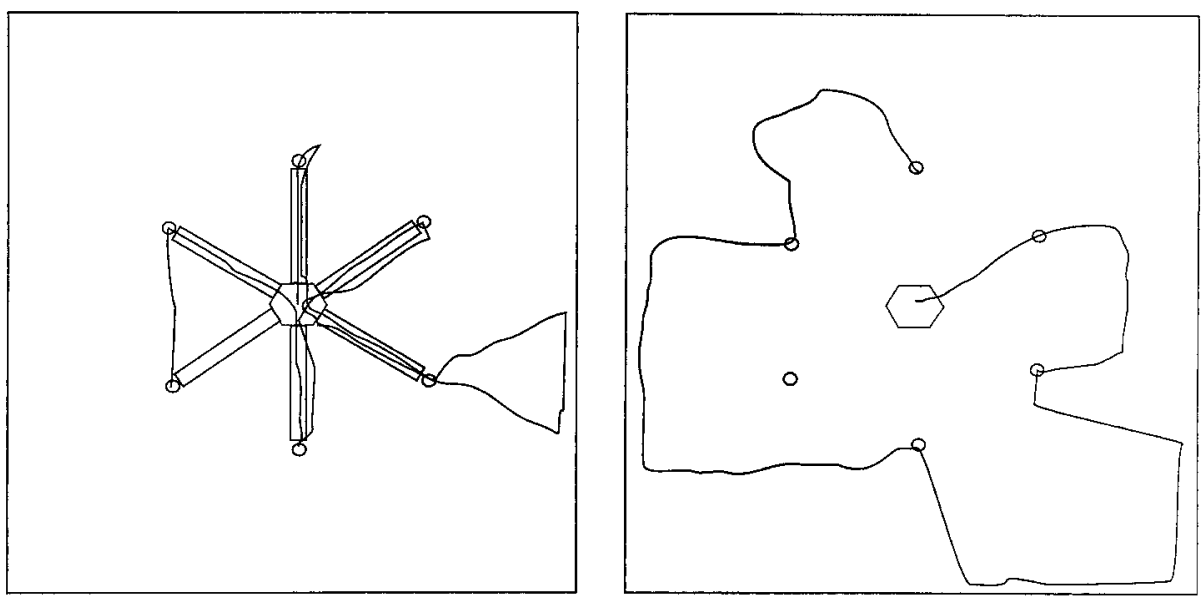

Figure 2. Modal path of Norway rats over the last 2 days of training (Days 11-12) in (left) a standard six-arm floor radial arm maze (RAM) and (right) a standard six-arm floor RAM without arms (data are from Hoffman, Timberlake, Leffel, \& Gont, 1999).

could establish and maintain such trails. Furthermore, trails are no protection from predators in such an open environment. A strict central-place foraging tactic appears unlikely even if the kangaroo rats were to treat each food cup as a patch, because they tend to move between multiple patches prior to returning to their burrows (Thompson, 1982).

The purpose of Experiment 2 was to use a floor RAM to investigate tactics used by desert kangaroo rats in efficiently searching the arms of a RAM. To allow us to precisely compare the behavior of Norway rats with that of Kangaroo rats, we used the same six-arm floor RAM as that used by Hoffman et al. (1999) and Timberlake et al. (1999). We established two configurations. The first consisted of a central platform with six arms radiating out, each arm leading to a food cup. The second configuration maintained the relationships of the central platform and the food cups, but all the arms were removed. The first and third experimental conditions used the standard floor RAM condition, whereas the second condition removed the arms. If the foraging tactic of kangaroo rats is similar to that of Norway rats, removal of the arms should be disrupting, and the behavior of the kangaroo rats should resemble that shown by the Norway rats in Figure 2.

On the other hand, finding distance-minimizing and/or meandering tactics would support an ecological analysis of radial arm behavior in the desert kangaroo rat. Furthermore, which tactic is dominant may tell us something of how the animal relates the environment of the RAM to its ecological foraging environment. If the kangaroo rats predominantly meander in this experiment, we would assume that they perceive the entire set of food cups as a single patch. If they show distance minimizing, they might be treating each food cup as a separate patch.

\section{Method}

Subjects. The subjects and housing were the same as those in Experiment 1 .
Apparatus. A six-arm radial maze constructed of wood with fiberboard (Masonite) edges was placed on the floor of the experimental room. The six-arm maze was used in order to allow us to compare kangaroo rat data with those of the Norway rats used by Hoffman et al. (1999). Testing was conducted in the same room as that in Experiment 1. A clear Plexiglas vertical divider was positioned in the room to create a roughly square $3.25 \times 3.66 \mathrm{~m}$ arena in which the maze was centered. The six arms radiated from a hexagonal center, with a $60^{\circ}$ angle between adjacent arms. The center measured $45 \mathrm{~cm}$ from one point to the opposite point of the hexagon; each arm was $13 \mathrm{~cm}$ wide and $70 \mathrm{~cm}$ long. The center and arms were $1.9-\mathrm{cm}-$ thick wood painted gray. Attached to the side and end of each arm was a 3.8-cm-high fiberboard border that extended $1.6 \mathrm{~cm}$ above the surface of the arm. The center also had 3.8-cm borders where the arms did not contact it. A hexagonal piece of clear Plexiglas was mounted $15 \mathrm{~cm}$ above the center platform and was covered with parallel, equally spaced, 1.2-cm strips of black construction paper so that only $50 \%$ of the area of the top was clear. The striped top for the center platform provided some potential cover for the foraging animal. A ceramic food cup, $7.5 \mathrm{~cm}$ in diameter and $5 \mathrm{~cm}$ tall, was placed on the floor at the end of each arm.

Procedure. Each kangaroo rat was again tested one trial a day: 12 days in the RAM1 (standard floor-RAM) condition, 12 days in the no-arm condition, and 6 days in the RAM2 (return to the standard floor-RAM) condition. Two $2 \times 2 \mathrm{~cm}$ pieces of lettuce were placed in each food cup on each trial. At the beginning of each trial, the kangaroo rat was placed in the center of the maze, oriented away from the experimenter. During the trial, the experimenter traced the travel pattern that the kangaroo rat followed on a data sheet on which the outline of the six-arm floor maze was centered in a rectangle representing the $3.66 \times 3.25 \mathrm{~m}$ arena. The rectangle was divided into a $9 \times 8$ grid of squares. To facilitate tracing the animal's path, we used masking tape on the floor of the actual arena to create the same $9 \times$ 8 grid of squares, $40.4 \mathrm{~cm}$ on a side. The experimenter separately noted the cups visited and the time elapsed at the end of the trial. A trial ended when the kangaroo rat had visited each of the six cups and one additional minute had elapsed, when it had visited food cups eight times, or at the end of $5 \mathrm{~min}$, whichever came first. At the end of the trial the kangaroo rat was gently corralled into a cardboard box, picked up by the experimenter, and returned to the carrier cage.

The counterbalancing of order of running was the same as that in Experiment 1 . To decrease the likelihood that the kangaroo rats' behavior would be influenced by odor cues left on the maze and on the floor of the room, the experimenter thoroughly wiped down the 
maze arms and central platform and mopped the floor, using RoccalD (a disinfectant, detergent, and deodorant) before each set of 4 kangaroo rats was tested.

Method of analysis. To describe how the kangaroo rats distributed their movement in the arena containing the floor RAM and to allow comparison with the behavior of Norway rats on the same apparatus, we categorized travel as either arm following, travel in the open, or wall following, using the criteria of Hoffman et al. (1999). In the RAM1 and RAM2 conditions, arm following included travel on or parallel to the arm (within $5 \mathrm{~cm}$ of the arm) for a distance of greater than $15 \mathrm{~cm}$. These criteria were assessed by laying a grid over the data tracing that divided each data square into four analysis squares, the side of each analysis square corresponding to $20.2 \mathrm{~cm}$ in the arena. Five centimeters corresponded to one fourth of the side of the analysis square, and $15 \mathrm{~cm}$ corresponded to three fourths of the side of the square. Scoring was done by coders blind to the expectations of the experimenter and was spot-checked initially for agreement. Arm following also included travel across the center of the maze if the kangaroo rat's travel continued through the center. Wall following included travel parallel to the wall and within $30 \mathrm{~cm}$ (1.5 grid squares) of the wall. Those parts of the kangaroo rats' locomotion that fit in neither the arm-following nor the wall-following category were included in the travel-in-the-open category.

We arrived at measurements of percentage of travel arm following, wall following, and open travel by counting the number of analysis squares traveled along an arm, along a wall, and in the open on each data sheet. The arm-following, open-travel, and wall-following portions of each day's travel were counted separately two times, and the mean was taken as the absolute distance of arm following, open travel, or wall following for that trial. The absolute distance traveled was totaled, and the percentage of arm following, open travel, and wall following was calculated for each trial for each kangaroo rat. The same basic categories and procedures were used in measuring behavior in the no-arm condition. Because locomotion continued to be recorded on data sheets that contained the representation of the maze arms, as well as the center platform, and the food cups, we used the same grid of analysis squares and defined behavior in the same way.

We analyzed two variables to assess the extent to which kangaroo rats traveled in an optimal route or a central-place foraging route. The most energetically efficient route that a kangaroo rat could take was to travel from the center of the maze to the first cup and then di- rectly between cups for the remaining five cups. We counted the number of instances per trial in which a kangaroo rat traveled from one cup to the next in the open (not following along an arm or along a wall) to assess how similar the travel pattern of our kangaroo rats was to that of an ideal distance minimizer. We termed moving directly from one cup to the next direct travel. To assess whether the kangaroo rats were showing a central-place foraging route, we counted the number of times that the kangaroo rats crossed the center of the maze. An ideal central-place forager beginning on the central platform would cross the center five times during each trial.

Chance, calculated with the same formula as that for Experiment 1, is 3.99 for an elevated six-arm maze. Because kangaroo rats on the floor RAM can leave the arms, chance is actually much lower than 3.99 because, since the animal does not have to return to the central platform, the food cups are not equally distant for the choice. We chose to use the value of 3.99 for our statistical tests for the RAM1, no-arm, and RAM2 conditions, however, to ensure a conservative estimate of chance. Finally, we again counted the percentage of visits to an adjacent arm, to assess whether the kangaroo rats were using a response algorithm for all the experimental conditions.

Dependent variables were analyzed using $t$ tests and analyses of variance (ANOVAs). Data were combined into two-trial blocks for analysis. One-group, one-tailed $t$ tests were conducted when the performance of kangaroo rats was compared with chance or with the expected value for distance minimizing or central-place foraging. Tests with probability levels less than .05 are reported as significant. ANOVAs were conducted comparing within-subjects performance over trials in each of the RAM1, no-arm, and RAM2 conditions. Additional ANOVAs compared the average of the last two trials across the RAM1, no-arm, and RAM2 conditions.

As was noted above, the maze and floor of the room were cleaned before each of the two groups of kangaroo rats was tested, to remove odor cues that might accumulate. To determine whether odors left on the maze and floor by kangaroo rats that ran early in each group had an effect on the foraging behavior of the kangaroo rats tested later in each group, we conducted ANOVAs on all dependent variables, comparing early kangaroo rats (the first 2 kangaroo rats in each group) with late kangaroo rats (the last 2 kangaroo rats in each group). There were no significant differences between kangaroo rats tested early and late in each group of 4 on any of the measures in any of the conditions.
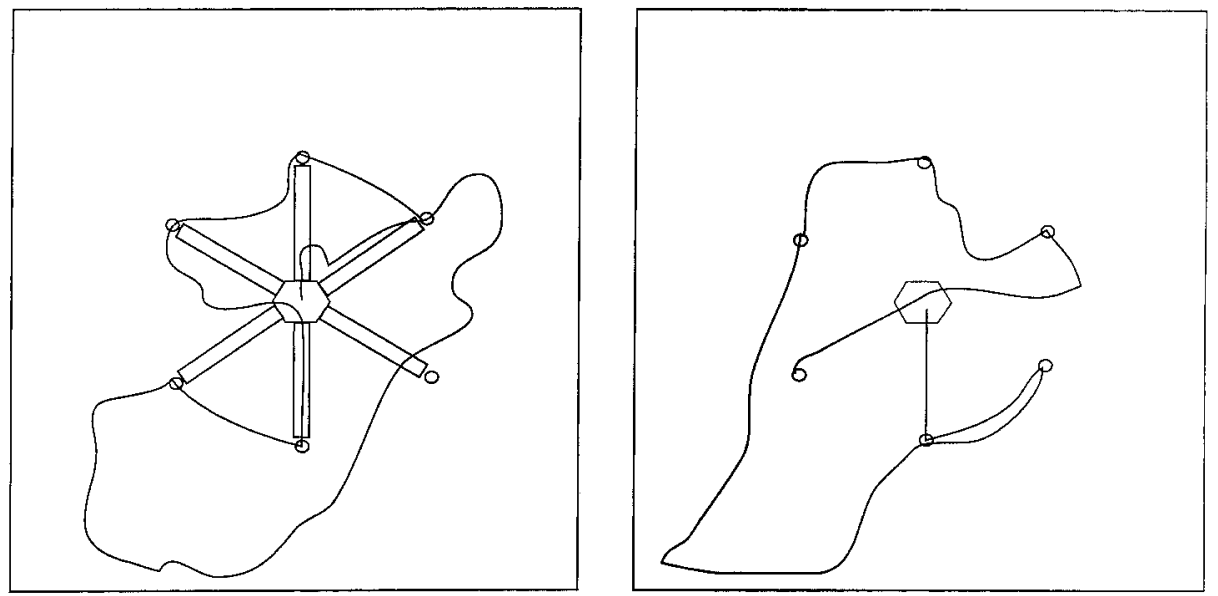

Figure 3. Modal path of kangaroo rats over the last 2 days of training (Days 11-12) in (left) a standard six-arm floor radial arm maze (RAM) and (right) a standard six-arm floor RAM without arms (data are from Experiment 2). 


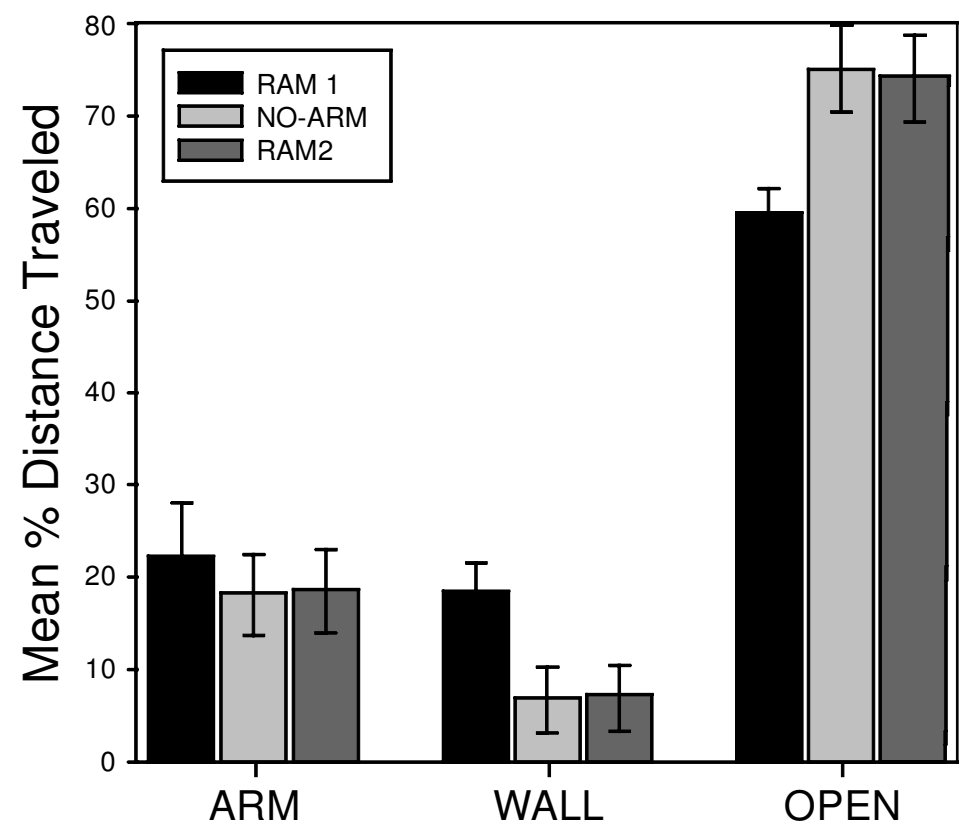

Figure 4. Mean percentage of distance traveled that occurred along maze arms, along walls, and in the open during the last two trials of the standard floor radial arm maze (RAM) condition (RAM1, Days 11-12), the arm removal condition (no-arm, Days 11-12), and the return to the standard floor RAM condition (RAM2, Days 3-4) (data are from Experiment 2).

\section{Results}

Figure 2 shows the modal asymptotic locomotor pattern of Norway rats on a floor RAM, with and without arms (Hoffman et al., 1999). Figure 3 shows the modal asymptotic pattern of our desert kangaroo rats exposed to the same conditions. A visual comparison of the figures indicates a considerable difference in the patterns of locomotion of the two species. In the presence of maze arms, the Norway rats predominantly traveled along them, whereas the kangaroo rats tended to ignore the arms and circle around the food cups. Removing the maze arms sent the rats along the walls of the arena but had very little effect on the locomotion of the kangaroo rats. Figure 4 provides quantitative support for the relative indifference of the kangaroo rats to maze arms by showing very little difference in the asymptotic mean percentage of total distance traveled in the open, along maze arms, and along arena walls in the RAM1, no-arm, and RAM2 conditions.

Search tactics. The tactic that the kangaroo rats approximated most closely was that of distance minimizing. Thus, Figure 5 shows that by far the greatest percentage of distance traveled was spent in the open (away from the walls, but not along the arms). This stands in marked contrast with the trail-following tactic of Norway rats, which traveled predominantly along maze arms and along walls when arms were absent (see Hoffman et al., 1999; Timberlake et al., 1999). Further evidence relevant to kangaroo rat tactics is shown in Figure 5. The top of Figure 5 shows low levels of center crossings (an index of central- place foraging) over the last two two-trial blocks. In contrast, the bottom of Figure 5 shows a marked increase in direct travel from one food cup to the next over the same period.

RAM1 condition. Additional data from the RAM1, noarm, and RAM2 conditions are shown in Table 2, averaged by two-trial blocks. In the RAM1 condition, despite not being confined to maze arms, the kangaroo rats tended to visit each food cup only once per trial, just as they did on the elevated RAM in Experiment 1 . The mean percentage of novel cups visited was $79.5 \%$ for kangaroo rats in RAM1, as compared with $81.1 \%$ on the elevated maze in Experiment 1. Table 2 also shows similarities between the present RAM1 condition and the elevated RAM of Experiment 1 in terms of the percentage of rewards taken over trials; the average percentage of rewards taken over the last two blocks of the RAM1 condition $(82.8 \%)$ was not distinguishable from the percentage of rewards taken over the last two blocks of elevated RAM performance in Experiment 1 (89.6\%).

There were other similarities between the RAM1 condition of Experiment 2 and the results of Experiment 1. The kangaroo rats in the RAM1 condition significantly decreased time to complete trials over two-trial blocks, just as in Experiment $1[F(5,35)=6.74, p<.01]$. The times for completing the floor RAM ranged from 111.4 to $219.8 \mathrm{sec}$, a range that overlapped the range of times for completing the elevated maze $(172.4-214.9 \mathrm{sec})$. Finally, the percentage of visits to an adjacent cup increased sig- 

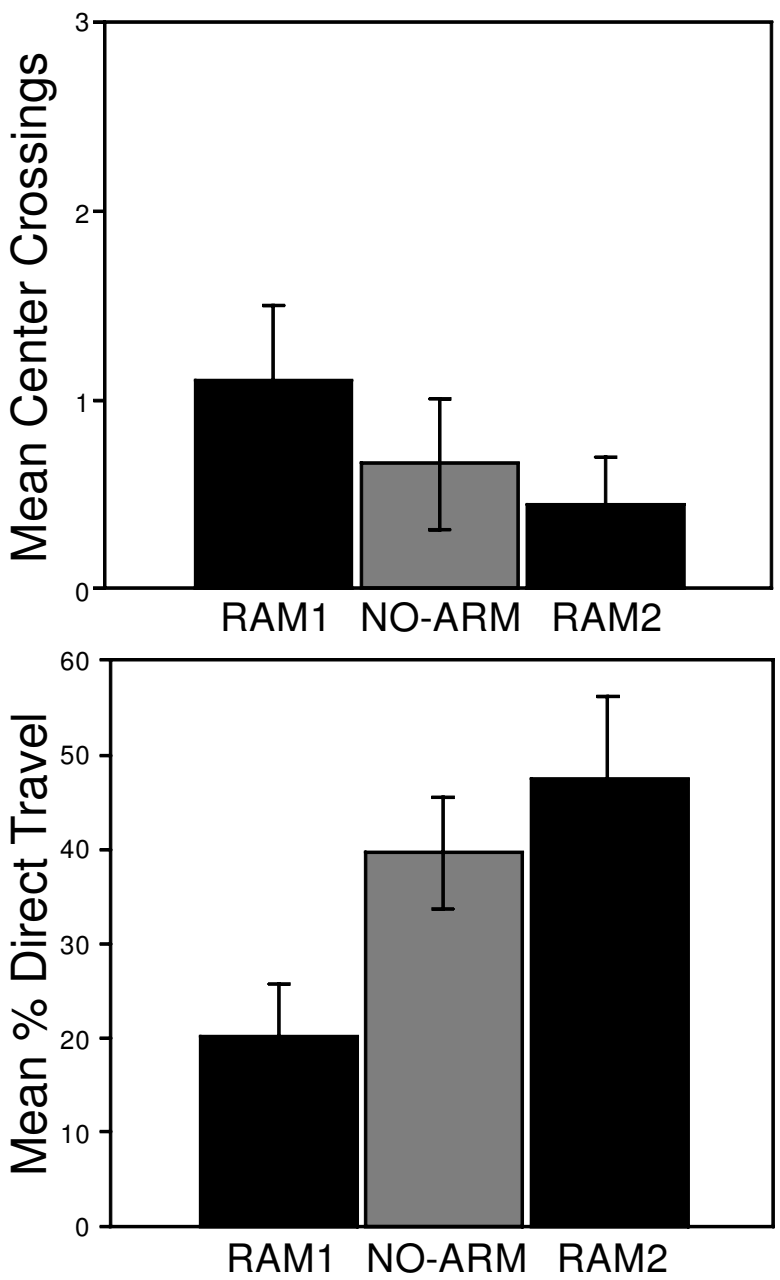

Figure 5. Top: mean center crossings for desert kangaroo rats (a measure of central-place foraging) averaged over the last two trials for the standard floor radial arm maze (RAM) condition (RAM1, Days 11-12), for the same maze configuration without arms (no-arms, Days 11-12), and for a return to the standard floor RAM condition (RAM2, Days 3-4). Bottom: mean percentage of direct travel between food cups for desert kangaroo rats (a measure of distance minimizing) for the last 2 days of the RAM1, no-arm, and RAM2 conditions (data are from Experiment 2).

nificantly over trials both in Experiment 1 and in the present RAM1 condition $[F(5,35)=6.15, p<.01]$. These similarities occurred despite the use of eight food cups in Experiment 1 and six food cups in Experiment 2. The major difference between elevated and floor RAM behavior was that the percentage of visits to an adjacent cup was considerably larger in the RAM1 floor RAM condition $(48.4 \%-20 \%)$. Apparently, not confining the animals to arms produced a much greater tendency to choose adjacent cups.

No-arm and RAM 2 conditions. As is also shown in Table 2, the removal of maze arms in the no-arms condition had almost no effect on performance of the kangaroo rats, relative to the previous RAM1 condition or the sub- sequent RAM2 condition. Measures showing no difference included the percentage of novel cups visited, the percentage of cups from which rewards were collected, and the time to complete a trial (all $p s>.10$ ). Furthermore, none of these measures changed significantly over blocks of days within the no-arm and RAM 2 conditions. The percentage of visits to adjacent arms did increase from the RAM1 to the no-arm condition $[F(1,14)=4.72, p<.05]$, but did not change from the no-arm to the RAM2 condition $[F(1,14)=0.91]$. As was also shown in the RAM1 condition, the percentage of visits to an adjacent cup in the no-arm condition increased over trial blocks $[F(1,14)=$ 3.89 ], with no change in the RAM2 condition.

\section{Discussion}

Desert kangaroo rats tested on the floor RAM performed nearly as accurately and efficiently as they had on the elevated maze, tending to visit each food cup only once per trial. There was a greater tendency to choose adjacent food cups, but this was not due to expression of a stereotyped response algorithm but, rather, to the increase in possibilities of orientation to and visiting of neighboring food cups. Like Norway rats, desert kangaroo rats did not show a strict central-place foraging tactic, but unlike Norway rats, they did not predominantly follow arms or walls. The percentage of travel along maze arms was low and stable across the RAM1, no-arm, and RAM2 conditions. Thus, the absence of the maze arms in the no-arm condition did not lead to a decrease in the amount of travel between the center of the maze and the food cups, as compared with the standard floor RAM condition.

The search patterns shown by the desert kangaroo rats appeared closest to a circular distance-minimizing tactic that was largely independent of the arms or walls but did include effective meandering. The meandering was not stereotyped but may have been mediated by the sight of nearby food cups, since there were few instances of doubling back on a path or crossing the center of the maze. The basic distance-minimizing path appeared remarkably similar from one trial to the next, although there was a significant increase over conditions in the percentage of direct travel and the percentage of adjacent cup choices. We conclude tentatively that the travel pattern of the kangaroo rats on a floor RAM is related to efficient search of a single patch defined by the food cups on the floor. However, it is still possible that the kangaroo rats treated each food cup as a separate patch.

\section{EXPERIMENT 3}

Experiment 2 showed that kangaroo rats, unlike Norway rats, do not employ a tactic of trail following in searching a floor RAM, nor do they appear to use a form of centralplace foraging. Instead, the kangaroo rats predominantly followed a distance-minimizing path, with some meandering thrown in. The purpose of Experiment 3 was to further investigate the roles of meandering and distance- 
Table 2

Mean Percentages of Novel Cups, Rewards Taken, and Visits to Adjacent Cups and Time to Complete a Trial on the Floor Radial Arm Maze (RAM) With Arms (RAM1, RAM2) and Without Arms (No-Arm)

\begin{tabular}{|c|c|c|c|c|c|c|c|c|c|c|c|c|c|c|c|}
\hline \multirow[b]{2}{*}{ Measure } & \multirow[b]{2}{*}{ Condition } & \multicolumn{2}{|c|}{ Block 1} & \multicolumn{2}{|c|}{ Block 2} & \multicolumn{2}{|c|}{ Block 3} & \multicolumn{2}{|c|}{ Block 4} & \multicolumn{2}{|c|}{ Block 5} & \multicolumn{2}{|c|}{ Block 6} & \multicolumn{2}{|c|}{ Mean } \\
\hline & & $M$ & $S E$ & $M$ & $S E$ & $M$ & $S E$ & $M$ & $S E$ & $M$ & $S E$ & $M$ & $S E$ & $M$ & $S E$ \\
\hline \multirow[t]{3}{*}{ Percentage of novel cups in six visits } & RAM1 & 78.6 & 4.6 & 77.7 & 2.1 & 80.2 & 3.1 & 82.1 & 2.5 & 79.2 & 3.1 & 83.1 & 3.5 & 80.2 & 1.7 \\
\hline & No-arm & 82.3 & 2.9 & 81.3 & 2.1 & 87.5 & 2.2 & 85.4 & 4.1 & 88.5 & 3.8 & 80.2 & 3.1 & 84.2 & 1.7 \\
\hline & RAM2 & 89.6 & 3.4 & 84.2 & 5.3 & 86.9 & 4.1 & - & - & - & - & - & - & 86.9 & 3.4 \\
\hline \multirow[t]{3}{*}{ Percentage of rewards taken } & RAM1 & 76.4 & 10.5 & 81.3 & 13.2 & 90.6 & 9.4 & 70.0 & 16.0 & 78.8 & 12.7 & 82.8 & 12.7 & 80.0 & 11.2 \\
\hline & No-arm & 78.8 & 14.2 & 70.3 & 15.3 & 72.9 & 13.1 & 78.8 & 15.2 & 77.5 & 14.9 & 59.4 & 14.9 & 72.9 & 13.9 \\
\hline & RAM2 & 72.8 & 14.6 & 82.3 & 12.8 & 83.3 & 12.6 & - & - & - & - & - & - & 79.5 & 13.0 \\
\hline \multirow[t]{3}{*}{ Percentage of visits to adjacent cups } & RAM1 & 27.1 & 6.6 & 39.8 & 6.7 & 39.8 & 8.2 & 44.2 & 7.2 & 46.9 & 6.3 & 54.5 & 2.5 & 42.0 & 4.9 \\
\hline & No-arm & 49.0 & 6.2 & 54.7 & 4.6 & 64.6 & 5.4 & 59.9 & 4.7 & 64.6 & 6.0 & 49.0 & 8.2 & 56.9 & 4.9 \\
\hline & RAM2 & 56.0 & 8.3 & 67.9 & 6.0 & 67.7 & 7.8 & - & - & - & - & - & - & 63.9 & 5.7 \\
\hline \multirow[t]{3}{*}{ Time to complete a trial $(\mathrm{sec})$} & RAM1 & 219.8 & 24.6 & 170.2 & 34.7 & 135.6 & 27.5 & 111.4 & 24.5 & 130.3 & 28.1 & 134.6 & 30.1 & 150.3 & 25.1 \\
\hline & No-arm & 121.3 & 21.7 & 114.9 & 31.4 & 79.4 & 14.9 & 96.9 & 16.8 & 82.6 & 11.6 & 105.8 & 29.2 & 100.1 & 19.2 \\
\hline & RAM2 & 129.9 & 31.6 & 105.4 & 33.3 & 86.8 & 23.6 & - & - & - & - & - & - & 107.4 & 27.3 \\
\hline
\end{tabular}

minimizing tactics as they relate to within-versus betweenpatch search.

If we consider floor RAM behavior as related to nicherelated foraging tactics, we may be able to modify the distribution of floor RAM cups to cast light on whether each food cup was a separate patch and the kangaroo rats traveled efficiently among them or whether each food cup was a food item within a patch and they foraged efficiently across the whole patch. To investigate this issue, we changed the symmetric circular distribution of food cups around the center platform used in Experiment 2 to one that could be characterized as two separate "patches" of cups, each consisting of four cups clumped at opposite ends of an elongated arena (see Figure 6). The choice platform remained in the center of the arena.
If this arrangement sufficiently mimics two separate patches, we would expect the animal to show meandering foraging within each patch and occasional beeline movement in traveling between them. In other words, the animal should show distance minimizing between the patches and a form of efficient meandering within them. On the other hand, if our arrangement does not represent separate patches to the kangaroo rats, we would expect a form of search closer to meandering across the entire set of cups, producing many trips between the two clumps of food cups. On the basis of the field observations of Thompson (1982; see his Figure 1), it is possible to argue either way, although stops to pouch seeds in patches in the open appear sufficiently close together to predict that the animals in our arena will see the two clumps of food cups as separate patches.

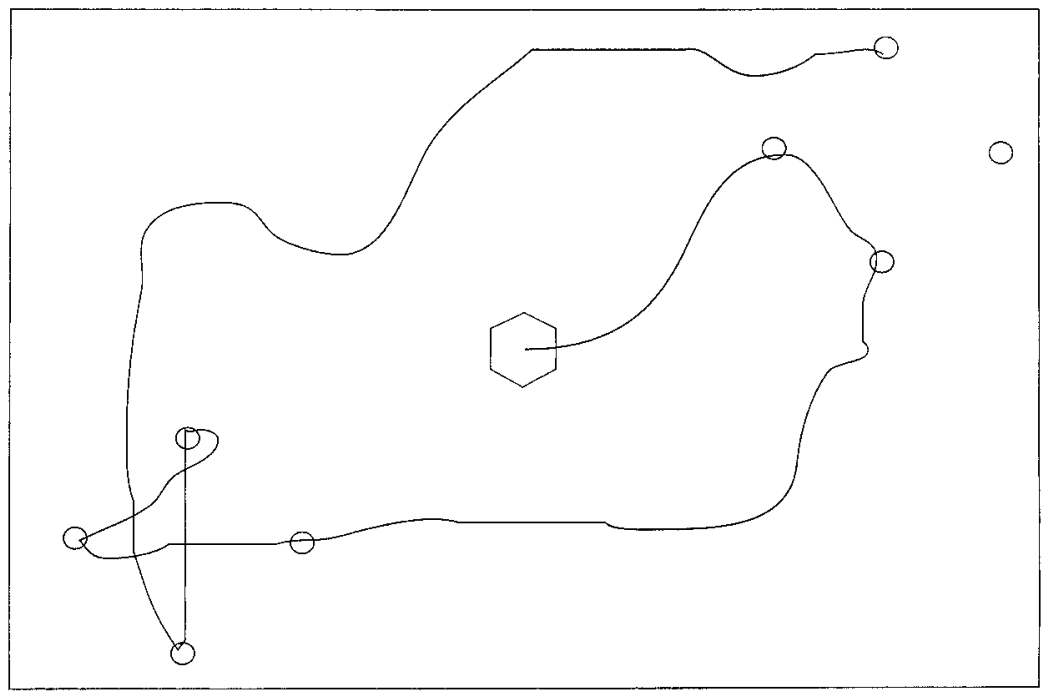

Figure 6. Modal path of desert kangaroo rats over the last 2 days of training (Days 11-12) in a condition with two separated "patches" of four food cups each (data are from Experiment 3). 

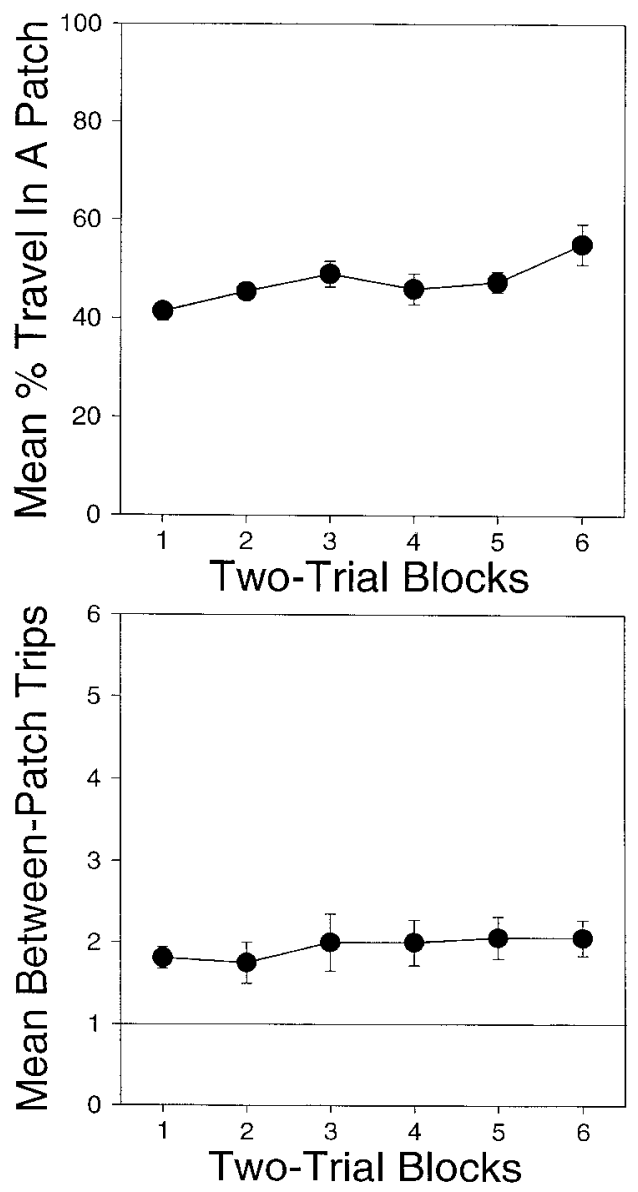

Figure 7. Top: mean percentage of total travel distance within a patch by two-trial blocks. Bottom: mean number of trips between patches by two-trial blocks (data are from Experiment 3 ).

\section{Method}

Subjects. The subjects and housing were the same as those in the previous experiments.

Apparatus. The center of the maze and the food cups were the same as those in Experiment 2. To create two potential patches, four food cups were placed at each of the opposite corners of an expanded $5.7 \times 3.25 \mathrm{~m}$ arena. Each member of a set of four food cups was at the corner of an implicit diamond shape, approximately $48 \mathrm{~cm}$ on a side and centered in an approximately $1.83-\mathrm{m}$ square in each corner (see Figure 6). Masking tape was used to divide the floor of the arena into $40.6-\mathrm{cm}$ squares, 8 along one dimension and 14 along the other. The maze platform was placed in the center of the arena, and each trial began with the kangaroo rat's being placed in the center, facing away from the experimenter.

Procedure. Each animal was run one trial per day for 12 days; the trial was terminated using the same criteria as those in Experiment 1, substituting food cups for arms. The experimenter traced the path of each animal on a data sheet divided into the same number of squares as those formed by the masking tape. The data sheet also contained a representation of the feeding cups and the central platform. The experimenter separately recorded visits to adjacent cups, mean percentage of rewards taken, mean percentage of novel cups in eight visits, and mean time to complete the trial.

Method of analysis. To describe how the kangaroo rats moved in the arena, we measured amount of within-patch and between- patch travel by overlaying each animal's data sheet with a gridded transparency. The grid consisted of 20.3-cm analysis squares, 16 along one dimension and 28 along the other. Within-patch travel was counted when an animal entered one of the 81 squares making up each patch (162 squares total). Between-patch travel was counted when the animal entered one of the other squares. These categories were counted twice and were combined to produce the total number of squares entered. The mean percentage of travel within a patch was calculated for each animal by dividing the number of within-patch analysis squares entered by the total number of analysis squares entered. Subtraction from $100 \%$ yielded between-patch travel.

We counted one additional variable: the number of trips between patches on each trial. The minimum number of trips between patches that an efficient kangaroo rat could travel is one if it locomoted from the center platform to one patch, visited all of the cups in that patch, and then traveled to the other patch to visit those cups. If the number of trips between patches is close to one, we can conclude that the kangaroo rats treated the patches as separate and thoroughly searched one before traveling to the other. The maximum number of trips between patches that a kangaroo rat could make was seven, provided it visited one new cup in each patch visit. If the number of trips between patches is close to seven, we can conclude that the kangaroo rats did not treat our two clumps as separate patches.

\section{Results and Discussion}

Figure 6 shows the modal search path for Trial Blocks $11-12$. It can be seen that the kangaroo rats foraged efficiently, largely treating the two sets of four food cups as separate patches. Figure 7 (top) shows the mean percentage of total travel distance occurring within the two possible patches (the 1.8-m squares containing four food cups). It can be seen that the percentage of total travel within one of the possible patches increased over trials $[F(5,35)=4.35, p<.05]$. As to travel between patches, the maximum number of trips that a kangaroo rat could make between the two groups of cups, provided it visited all eight cups, was seven. The minimum number of trips a kangaroo rat could make and still visit the majority of the cups was one. Figure 7 (bottom) shows that the number of trips between patches ranged from 1.3 to 2.8 , with a mean of 1.95. This number of trips was significantly less than seven $[t(7)=27.62]$ and significantly greater than one $[t(7)=5.18$, both $p$ s $<.01]$.

That kangaroo rats visited an average of 6.5 novel cups per trial and traveled an average of 1.95 trips between patches means that they typically collected food in two to three of the four cups in one patch before traveling to the other patch. Thus, the collection of food in each patch before leaving was modestly thorough. Furthermore, the number of trips between these potential patches was much closer to that of a complete distance-minimizing single trip than the central-place foraging or meandering maximum of 7 trips between patches per trial.

Table 3 provides additional data in terms of the same measures taken in Experiment 2 in the RAM1 and no-arm conditions averaged over two-trial blocks (compare with Table 2). The percentage of novel cups visited in six visits, the percentage of rewards taken, the percentage of visits to adjacent cups, and the time to complete a trial all fell within the ranges recorded for Experiment 2, RAM1 and no-arm conditions. The percentage of novel cups out of a 
Table 3

Mean Percentages of Novel Cups, Rewards Taken, and Visits to Adjacent Cups and Time to Complete a Trial in the Two-Patch Floor Condition (Experiment 3)

\begin{tabular}{|c|c|c|c|c|c|c|c|c|c|c|c|c|c|c|}
\hline \multirow[b]{2}{*}{ Measure } & \multicolumn{2}{|c|}{ Block 1} & \multicolumn{2}{|c|}{ Block 2} & \multicolumn{2}{|c|}{ Block 3} & \multicolumn{2}{|c|}{ Block 4} & \multicolumn{2}{|c|}{ Block 5} & \multicolumn{2}{|c|}{ Block 6} & \multicolumn{2}{|c|}{ Mean } \\
\hline & $M$ & $S E$ & $M$ & $S E$ & $M$ & $S E$ & $M$ & $S E$ & $M$ & $S E$ & $M$ & $S E$ & $M$ & $S E$ \\
\hline Percentage of novel cups in 8 visits & 76.6 & 3.7 & 75.0 & 4.1 & 82.3 & 3.3 & 89.7 & 2.3 & 82.8 & 3.3 & 83.6 & 2.6 & 81.7 & 2.2 \\
\hline Percentage of rewards taken & 79.2 & 14.0 & 88.5 & 12.7 & 75.5 & 14.7 & 67.2 & 15.6 & 81.3 & 13.2 & 78.1 & 14.5 & 78.3 & 13.5 \\
\hline Percentage of visits to adjacent cups & 74.0 & 5.3 & 73.3 & 5.4 & 57.6 & 9.6 & 60.4 & 7.4 & 63.6 & 9.0 & 57.9 & 5.7 & 64.4 & 5.7 \\
\hline Time to complete a trial $(\mathrm{sec})$ & 128.7 & 15.6 & 107.0 & 23.3 & 124.9 & 31.4 & 126.5 & 30.5 & 91.7 & 21.2 & 96.0 & 19.0 & 112.5 & 22.1 \\
\hline
\end{tabular}

maximum of six visits was significantly above chance $[t(7)=7.17]$.

In short, although the kangaroo rats did not perfectly minimize the distance traveled, in that they most often switched patches more than once, they nearly always searched more than one food cup on entry to a patch, and they rarely switched patches more than twice. We conclude that the kangaroo rats treated the two groups of cups as separate patches. We also conclude that the relative proximity of food items is an important determinant of how kangaroo rats divide food sources into patches and, thus, is an important determinant of its pattern of locomotor search.

\section{GENERAL DISCUSSION}

Desert kangaroo rats, like Norway rats, performed efficiently on an elevated RAM, apparently using spatial memory to avoid revisits to arms within a trial. Unlike Norway rats, they initially collected and pouched only about half of the rewards (lettuce pieces) and never appeared to actually ingest the rewards on the maze. It is worth pointing out that the kangaroo rats' ability to perform efficiently on a RAM without promptly ingesting the reward may not be unique. Female Norway rats placed on an unbaited maze searched all the arms almost as efficiently as rats performing on a baited maze (Timberlake \& White, 1990). Our procedures do not rule out the possibility that the kangaroo rats were secondarily reinforced by inspecting the lettuce in the food cup, but neither primary nor secondary reinforcement does a complete job of explaining their behavior. For both species, efficient RAM search appears to be based on general locomotor search mechanisms engaged by the feeding system and the environment (Timberlake, 1983, 2002; Tinsley et al., 2001).

The similarity between Norway rats and desert kangaroo rats extended also to their efficiency in visiting the food cups of an RAM placed flat on the floor (a floor RAM), but it did not include the locomotor tactics they used in searching the floor RAM. Norway rats solved the floor maze largely by following arms (Hoffman et al., 1999; Timberlake et al., 1999), apparently a variant of the trail following rats predominantly show in field environments (Calhoun, 1962; Telle, 1966). In contrast, the desert kangaroo rats traveled considerably more in the open than along the maze arms and appeared to approximate a distanceminimizing tactic much more closely than did the Norway rats. Furthermore, when the arms of the maze were eliminated, the Norway rats moved along the walls between food cups, whereas the kangaroo rats followed roughly the same distance-minimizing paths that they did when the arms were in place.

In Experiment 3, we gained potential insight into how kangaroo rats spatially define a patch. When we separated the food cups in our arena into two groups of four, each equidistant from the central platform and on opposite sides, the animals appeared to treat them as separate patches. They efficiently meandered among the cups in a patch and, on average, traveled directly between patches fewer than two times a trial. This result raises the possibility that concentrations closer together than $10 \mathrm{~m}$ may still be treated as separate patches, a possibility that is compatible with observations of kangaroo rats foraging on patches in the open (Thompson, 1982). Whether an arena in the laboratory corresponds to an open-field environment is not clear.

An ecological approach to the study of learning, such as behavior systems (Timberlake, 1983, 1990, 2001b, 2002), argues that animals perform well on common foodreinforced laboratory tasks such as the elevated RAM, because experimenters have analytically and inductively tuned the task to map onto the niche-related mechanisms determining natural foraging behavior. Tuning refers to the iterative process whereby experimenters modify the apparatus and procedures to produce more reliable and vigorous behavior. For example, Timberlake (2002) summarized evidence that several types of laboratory maze have been tuned to the characteristics of the Norway rats.

Given the apparent success of experimenters in tuning laboratory paradigms to make contact with niche-related mechanisms, it should prove possible to use manipulations of laboratory environments to analyze the determinants of field-related behavior (e.g., Balsam \& Silver, 1994; Timberlake, 1990, 2002; Tinbergen, 1951). Thus, in the present research, we were able to predict that kangaroo rats would be much less likely than Norway rats to use maze arms as trails to locate food items or to use arena walls when maze arms were missing. It should follow that kangaroo rats would not be disoriented like Norway rats when maze arms were misaligned with food cups (Roche \& Timberlake, 1998) nor as perturbed in their behavior as Norway rats by the clipping of their whiskers. In the present research, we also provided initial data on how kangaroo rats may define patches. 
There are a variety of reasons to expect a divergence in tactics between domestic Norway rats and kangaroo rats. Norway rats are highly social animals that spend the majority of their time above ground locomoting on trails established by the colony (Telle, 1966). These trails provide a social linkage within a colony in terms of food sources, harborage sites, and "message boards" constructed by urine marking (Calhoun, 1962). In contrast, solitary desert kangaroo rats, although showing some constant paths around the entrances to their burrows, appear to use landmarks in visiting foraging patches, a tactic in keeping with their solitary, aggressive nature (Congdon, 1974; Stern, 1980) and with the difficulty of maintaining foraging trails in a sandy and wind-blown environment. Thus, at the least, trail following in desert kangaroo rats should be considerably less important, whereas distance minimizing should be relatively more important than for Norway rats.

Finally, caution is important in relating ecological behavior to laboratory environments and experimenter tuning. The appropriate level of concern with behavior should be in terms of underlying niche-related mechanisms and their evolution and expression. It is the expression of the mechanisms in a particular environment that has been the basis of selection. For example, foraging patterns observed in the field are always a function of the interaction of task demands and mechanisms. Thus, although experimenter tuning clearly can make contact with ecological behavior, there may be multiple mechanisms involved, and they may not be engaged in the laboratory in the same way as they are in ecological niches. Thus, foraging patterns based on the same set of mechanisms may be different in different laboratory environments, just as they may differ in alternative field environments, depending on the distribution of food, the type of vegetation and terrain, the animal's previous experience, and such variables as motivation, predator pressure, and reproductive state.

\section{REFERENCES}

Balsam, P. D., \& Silver, R. (1994). Behavioral change as a result of experience: Toward principles of learning and development. In J. A. Hogan \& J. J. Bolhuis (Eds.), Causal mechanisms of behavioral development (pp. 327-357). Cambridge: Cambridge University Press.

CAlHoun, J. B. (1962). The ecology and sociology of the Norway rat. Bethesda, MD: U.S. Department of Health, Education and Welfare (Publication No. 1008).

Congdon, J. (1974). Effect of habitat quality on distributions of three sympatric species of desert rodents. Journal of Mammalogy, 55, 659662.

Hailman, J. P. (1967). The ontogeny of an instinct: The pecking response in chicks of the laughing gull (Laurus artricilla $\mathrm{L}$.) and related species. Behaviour, $\mathbf{X V}$ (Suppl.), 1-159.

HeARST, E., \& Jenkins, H. M. (1974). Sign-tracking: The stimulusreinforcer relation and directed action. Austin, TX: Psychonomic Society.

Hoffman, C. M., Timberlake, W., Leffel, J., \& Gont, R. (1999). How is radial arm maze behavior related to locomotor search tactics? Animal Learning \& Behavior, 27, 426-444.

JACOBS, L. (1992). Memory for cache locations in Merriam's kangaroo rats. Animal Behaviour, 43, 585-593.
KotLER, B. (1984). Risk of predation and the structure of desert rodent communities. Ecology, 65, 689-701.

KotLER, B. (1985). Owl predation on desert rodents which differ in morphology and behavior. Journal of Mammalogy, 66, 824-828.

LANGLey, C. M. (1994). Spatial memory in the desert kangaroo rat (Dipodomys deserti). Journal of Comparative Psychology, 108, 3-14.

Olton, D. S., Handelmann, G. E., \& Walker, J. A. (1978). Spatial memory and food searching strategies. In A. C. Kamil \& T. D. Sargent (Eds.), Foraging behavior: Ecological, ethological and psychological approaches (pp. 333-354). New York: Garland.

Olton, D. S., \& SAmuelson, R. J. (1976). Remembrance of places passed: Spatial memory in rats. Journal of Experimental Psychology: Animal Behavior Processes, 2, 97-116.

Rebar, C. E. (1995). Ability of Dipodomys merriami and Chaetodipus intermedius to locate resource distributions. Journal of Mammalogy, 76, 437-447.

Reichman, O. J. (1984). Spatial and temporal variation of seed distribution in Sonoran Desert soils. Journal of Biogeography, 11, 1-11.

Reichman, O. J., \& Oberstein, D. (1977). Selection of seed distribution types by Dipodomys merriami and Perognathus amplus. Ecology, 58, 636-643.

Roche, J. P., \& Timberlake,W. (1998). The influence of artificial paths and landmarks on the foraging behavior of Norway rats (Rattus norvegicus). Animal Learning \& Behavior, 26, 76-84.

STERN, B. (1980). Habitat partitioning in two species of kangaroo rat (Rodentia: Heteromyidae): Dipodomys deserti and Dipodomys merriami. Unpublished doctoral dissertation, University of California, Berkeley.

Telle, H. J. (1966). Beitrage zur Kenntnis der Vehaltensweise von Ratten, vegleichend dargestellt bei, Rattus norvegicus und Rattus rattus. Zeitschrift für Angewandte Zoologie, 48, 97-129.

Thompson, S. D. (1982). Microhabitat utilization and foraging behavior of bipedal and quadrupedal heteromyid rodents. Ecology, 63, 1303-1312.

Timberlake, W. (1983). Appetitive structure and straight alley running. In R. L. Mellgren (Ed.), Animal cognition and behavior (pp. 165221). New York: North-Holland.

Timberlake, W. (1990). Natural learning in laboratory paradigms. In D. A. Dewsbury (Ed.), Contemporary issues in comparative psychology (pp. 31-54). Sunderland, MA: Sinauer.

TimberlaKe, W. (2001a). Integrating niche-related and general process approaches in the study of learning. Behavioural Processes, 54, 79-94.

Timberlake, W. (2001b). Motivational modes in behavior systems. In R. R. Mowrer \& S. B. Klein (Eds.), Handbook of contemporarylearning theories (pp. 155-209). Mahwah, NJ: Erlbaum.

Timberlake, W. (2002). Niche-related learning in laboratory paradigms: The case of maze behavior in laboratory rats. Behavioural Brain Research, 134, 355-374.

Timberlake, W., Leffel, J., \& Hoffman, C. M. (1999). Stimulus control and function of arm and wall travel by rats on a radial arm floor maze. Animal Learning \& Behavior, 27, 445-460.

Timberlake, W., \& White, W. (1990). Winning isn't everything: Rats need only food deprivation not food reward to traverse a radial arm maze efficiently. Learning \& Motivation, 21, 153-163.

Tinbergen, N. (1951). The study of instinct. New York: Oxford University Press.

Tinbergen, N., \& Perdeck, A. C. (1950). On the stimulus situation releasing the begging response in the newly hatched herring gull chick (Larus argentatus Pont.). Behaviour, 3, 1-39.

Tinsley, M. R., Rebec, G. V., \& Timberlake, W. (2001). Facilitation of efficient search of an unbaited radial-arm maze in rats by D1, but not D2, dopamine receptors. Pharmacology, Biochemistry \& Behavior, 70, 181-186.

(Manuscript received April 24, 2002; revision accepted for publication July 18,2002 .) 